\title{
TLS in the Wild: An Internet-wide Analysis of TLS-based Protocols for Electronic Communication
}

\author{
Ralph Holz*, Johanna Amann ${ }^{\ddagger}$, Olivier Mehani ${ }^{\dagger}$, Matthias Wachs ${ }^{\S}$, Mohamed Ali Kaafar ${ }^{\dagger}$ \\ *University of Sydney, Australia, Email: ralph.holz@sydney.edu.au \\ ${ }^{\dagger}$ Data61/CSIRO, Sydney, Australia, Email: name.surname@data61.csiro.au \\ †ICSI, Berkeley, USA, Email: johanna@icir.org \\ $\S$ Technical University of Munich, Germany, Email: wachs@net.in.tum.de
}

\begin{abstract}
Email and chat still constitute the majority of electronic communication on the Internet. The standardisation and acceptance of protocols such as SMTP, IMAP, POP3, XMPP, and IRC has allowed to deploy servers for email and chat in a decentralised and interoperable fashion. These protocols can be secured by providing encryption with TLS-directly or via the STARTTLS extension. X.509 PKIs and ad hoc methods can be leveraged to authenticate communication peers. However, secure configuration is not straight-forward and many combinations of encryption and authentication mechanisms lead to insecure deployments and potentially compromise of data in transit. In this paper, we present the largest study to date that investigates the security of our email and chat infrastructures. We used active Internet-wide scans to determine the amount of secure service deployments, and employed passive monitoring to investigate to which degree user agents actually choose secure mechanisms for their communication. We addressed both client-to-server interactions as well as server-to-server forwarding. Apart from the authentication and encryption mechanisms that the investigated protocols offer on the transport layer, we also investigated the methods for client authentication in use on the application layer. Our findings shed light on an insofar unexplored area of the Internet. Our results, in a nutshell, are a mix of both positive and negative findings. While large providers offer good security for their users, most of our communication is poorly secured in transit, with weaknesses in the cryptographic setup and especially in the choice of authentication mechanisms. We present a list of actionable changes to improve the situation.
\end{abstract}

\section{INTRODUCTION}

Despite the rise of mobile messaging and some more centralised, newer communication platforms, two forms of electronic, (nearly) instant messaging still remain dominant on the public Internet: email and chat. Of the two, email is the most pervasive form of communication ever, with over 4.1 billion accounts in 2014, predicted to reach over 5.2 billion in 2018 [11]. As for chat, the most widely used standard-based networks are IRC group chats and the XMPP instant messaging and multi-user conferencing network.

\footnotetext{
* The work was carried out during the first author's time at Data61/CSIRO.
}

Permission to freely reproduce all or part of this paper for noncommercial purposes is granted provided that copies bear this notice and the full citation on the first page. Reproduction for commercial purposes is strictly prohibited without the prior written consent of the Internet Society, the first-named author (for reproduction of an entire paper only), and the author's employer if the paper was prepared within the scope of employment.

NDSS ’16, 21-24 February 2016, San Diego, CA, USA

Copyright 2016 Internet Society, ISBN 1-891562-41-X

http://dx.doi.org/10.14722/ndss.2016.23055
In their early days, email protocols such as SMTP, POP3, and IMAP were designed with no special focus on security. In particular, authentication in SMTP was introduced a while after the protocol's standardisation, initially as a way to fight spam. User agents started to move towards encryption and authenticated connections gradually, using the then-new SSL 3 and later the TLS protocols to protect the transport layer. SSL/TLS can provide authentication, integrity, and confidentiality. Where SSL/TLS is not used, user credentials may be transmitted in plaintext, with no protection against eavesdropping, and message bodies can be tampered with (unless end-to-end mechanisms like OpenPGP or S/MIME are used, which is a comparatively rare setup).

Although SSL/TLS support mutual authentication, the most common usage pattern in the context of email and chat is unilateral authentication: only the responder of a communication is authenticated on the transport layer. The primary reason for this is the protocols' reliance on an X.509 Public Key Infrastructure (PKI) for authentication purposes ${ }^{1}$ and the subsequent need for client certification, an operation that is expensive in practice, introduces much administrative overhead, and often also requires user education. In most cases, initiators are authenticated on the application layer instead, i.e., by mechanisms that are specific to the application layer protocol in question. Passwords schemes are the most common choice, although any mechanism that is supported by both initiator and responder is possible. Different password schemes offer varying levels of security-e.g., the password may be sent without further protection over the SSL/TLS channel, or a challenge-response mechanism like CRAM, or even SCRAM, may be used. The latter is particularly elegant as it does not require the responder to store the actual password, nor is the password ever sent over the connection. The choice of password scheme has a profound influence on security in case of missing authentication on the level of SSL/TLS.

The proper in-band authentication of the responder is a key element in SSL/TLS. X.509 certificates are used for this purpose. These are issued by so-called Certificate Authorities (CAs), which are trusted parties whose trust anchors (so-called root certificates) are shipped with common software (e.g., operating systems, browsers, mail clients, ...). Unfortunately, it is known today that X.509 PKIs often suffer from poor deployment practices. Holz et al. [24] were the first to show this in a largescale, long-term study for the Web PKI. Durumeric et al. [7]

\footnotetext{
${ }^{1}$ Variants of TLS that support other forms of authentication have been standardised, but seem to be rarely used.
} 
later extended the study to all Internet hosts, confirming the earlier findings. However, no such work exists for the electronic communication protocols on which we rely every day.

In this paper, we present the largest measurement study to date that investigates the security of SSL/TLS deployments for email and chat. Based on our findings, we derive recommendations to achieve better overall security. We employ both active Internet-wide scans as well as passive monitoring. Active scans are used to characterise global server installations, i.e., how servers are configured to act as responders in a SSL/TLS connection. Passive monitoring allows us to investigate the actual security parameters in use when initiators establish SSL/TLS connections.

From 2015-06-30 through 2015-08-04, we actively scanned the IPv4 address space (3.2B routable addresses), with one scanning run for each protocol we analysed. We connected to the standard ports for the considered protocols: SMTP/STARTTLS, SMTPS, SUBMISSION, IMAP/STARTTLS, IMAPS, POP3/STARTTLS, and POP3S for email; for chat, we investigated IRC/STARTTLS, IRCS, XMPP/STARTTLS, and XMPPS. We performed complete SSL/TLS handshakes. This allowed us to establish a list of current deployments (a total of more than 50M active ports), and collect certificates, cipher suite offers, and cryptographic parameters. Where applicable, we also sent application-layer messages to request the list of supported methods for authentication on the application layer. Orthogonally to this, nine days of passive monitoring (2015-07-29 through 2015-08-06) of a link serving more than 50,000 users showed more than $16 \mathrm{M}$ connections to about 14,000 different services. We captured the same set of SSL/TLS and authenticationrelated parameters from this monitoring data as we did for active scans. This allows us to compare usage by actual clients to the simple existence of a deployed service. As a reference and comparison point, we also considered HTTPS and traffic on port 443 in both active and passive measurements as the deployment of this protocol is particularly well understood.

We analysed this data to evaluate the security of connections and deployments. We considered the validity of the certificates and the practices of the issuing CAs, the quality of cryptographic parameters, software, and SSL/TLS versions, as well as the authentication methods. In a nutshell, we have both negative and positive findings to report. Considering active scans, we find that there is much room for improvement. For example, for the IMAPS servers that completed the TLS handshake, we report that just under $40 \%$ also had correct certificate chains deployed. We found such low rates for all protocols-the best-provisioned service was in fact SMTPS, where just over $40 \%$ of servers had valid certificate chains. SMTP/STARTTLS, which is used to forward emails between mail exchange servers, showed a rate of just $30 \%$. For chat, we found the best results for XMPPS in server-to-server forwarding: $27 \%$ of servers offered valid certificates.

When considering data from our passive monitoring and investigating connections rather than server deployments, the situation seems much better, at least at a first glance: the vast majority of connections is encrypted and uses valid certificates (with SMTP/STARTTLS again showing poorer numbers, however). This is due to the fact that large providers such as Gmail or Hotmail are properly configured and offer good security, and most connections go to these providers. However, we also found that it is common that the STARTTLS extension is not supported by servers that receive less connections. In these cases, connections are not encrypted at all. This is again particularly often true for email. This phenomenon suggests a likelihood that communication is often not sufficiently secured in transit between mail exchange servers, unless both sender and receiver are customers of large providers.

The rest of this paper is organised as follows. The next section presents background for SSL/TLS, PKI, and the studied protocols. It also gives an overview of related work. We describe our data collection methods and datasets in Section III and data analysis in Section IV. Based on our findings, we identify risks and threats in Section V. We suggest some pathways towards improving the situation before concluding in Section VI.

\section{BACKGROUND AND RELATED WORK}

\section{A. Standard messaging protocols}

The messaging protocols in common use today have been specified by the IETF over the years; use with SSL/TLS or the STARTTLS extension was added later. For example, the original RFC 821 for SMTP is from 1982, but the STARTTLS extension for SMTP was specified in 1999. Other protocols experienced similar organic development, and the result is a variety of ways in which SSL/TLS is used in email and chat.

a) Electronic Mail: Email relies on two sets of protocols: one for email transfer and one for retrieval. The Simple Mail Transfer Protocol (SMTP) [27] is the cornerstone of email distribution systems. Its primary purpose was message transfer: so-called Message Transfer Agents (MTAs) forward messages by establishing an SMTP session to the next MTA on the path to the destination, until they arrive at their final destination. SMTP is also used as a submission protocol ${ }^{2}$ : in a nutshell, user agents—e.g., 'email clients' such as Thunderbird—submit mails from a local computer for further delivery to a 'mail server' that is commonly operated by the user's service provider. 'Webmail' solutions such as GMail blur the distinctions between mail submission and mail transfer somewhat: they offer webhosted front-ends for mail composition; mail submission and mail transfer are handled entirely transparently on server-side. SMTP was initially operated on port 25 . Later, port 587 was specified to be used for message submission [16] by potentially authenticated submitters, in an attempt to differentiate between legitimate activity and spam. Nevertheless, port 25 still remains in use for both purposes, message transfer and submission.

Once at the destination server, email can be retrieved using either of two protocols. The Post Office Protocol (version 3, commonly referred to as POP3) [30] operates on port 110 and allows a remote client to download newly-received emails to a local mailbox. The Internet Message Access Protocol (version 4, commonly called IMAP) [4] uses port 143 and offers access, manipulation, and download of messages in a mailbox stored on the server side.

b) Chat and Instant Messaging: Instant chat is an old concept, which predates even the Web. Internet Relay Chat (IRC) [34] is a protocol that allows a number of IRC clients to connect to an IRC server and join so-called channels (chat

\footnotetext{
${ }^{2}$ This was possibly first made explicit in RFC 2476 [15].
} 
rooms) or have private conversations. Messages, especially on channels, are relayed between IRC servers. An oddity of IRC deployments is that server-to-server communication is implementation-dependent (despite a specification in [26]). Over time, this has led to IRC servers clustering into a number of distinct 'IRC networks'. While the official IANA port for clientto-server connections is 194, IRC is most commonly deployed on port 6667 instead [17], but other ports are also sometimes used. The ports for server-to-server communication are specific to the IRC network.

In the footsteps of the proprietary instant messaging (IM) networks of the late 1990s, the more general XML-based eXtensible Messaging and Presence Protocol (XMPP) was specified. Its core functionality is defined in RFC 6120 [39], IM extensions in RFC 6121 [40]. Further extensions exist. Similar to the SMTP infrastructure, a number of XMPP servers exchange messages on behalf of their users as part of the XMPP IM network ${ }^{3}$. The protocol uses port 5222 for client-toserver communication, and 5269 for server-to-server forwarding. XMPP, with or without proprietary extensions, is also used in non-federated enterprise or proprietary services ${ }^{4}$.

\section{B. $S S L / T L S$}

TLS 1.0 is the IETF-standardised version of SSL $3^{5}$. All versions before TLS 1.0, i.e., SSL 2 and SSL 3, are deprecated today. TLS is at version 1.2 and contains many critical fixes that remove weaknesses of previous versions. Version 1.3 is currently in the standardisation process. As SSL 3 and TLS 1.0 are very similar and a few pockets of SSL 3 use remain, we speak of SSL/TLS when our findings apply to both SSL 3 and TLS. All email and chat protocols can be used with SSL/TLS. In IMAP and POP3, only client-to-server communication occurs. SMTP and XMPP define both client-to-server and server-toserver communication patterns. For IRC, once again only the client-to-server pattern is properly defined.

There are two ways to negotiate an SSL/TLS session. The first is to use SSL/TLS directly. This requires a well-known port, i.e., assignment of a new, dedicated port by IANA. Application layer protocols that use this method are often indicated by adding a ' $\mathrm{S}$ ' at the end, e.g., HTTPS, IMAPS, etc. Clients that do not support SSL/TLS may still connect to the normal port. In server-to-server communication, the servers may use certificates to authenticate to each other (i.e., either unilateral or mutual authentication may be used). As stated in the introduction, in client-to-server communication it is common that only the server is authenticated; the client is authenticated later as part of the application layer protocol. In the case of SMTP, port 465 was initially defined for SMTPS, but was deprecated later [22] in favour of STARTTLS (see below). It is nevertheless still used. The dedicated ports for IMAPS and POP3S are 993 and 995, respectively. For IRCS, several exist [17], with 6697 being very commonly used for client connections. XMPP does not have standard ports for SSL/TLS, but ports 5223 and 5270 are prevalent for client-to-server and server-to-server communication, respectively.

\footnotetext{
${ }^{3}$ XMPP Instant Messaging was known as Jabber before its standardisation.

${ }^{4}$ E.g., HipChat uses a flavour of XMPP, as did the early Google Talk.

${ }^{5}$ SSL 3, originally created at Netscape, was never standardised by the IETF, but later captured in a historic RFC [13].
}

The second major way to use SSL/TLS is to connect with TCP on the normal port first and then upgrade the connection using a protocol-specific command. This method is commonly referred to as STARTTLS. The specifications in the RFCs commonly require clients to first query a server for STARTTLS support with a specific 'capability' command before trying to upgrade the connection [32]. The server can confirm an upgrade; the SSL/TLS handshake follows. This is specified for SMTP (particularly for SUBMISSION) in [20], in [32] for IMAP and POP3, and in [41] for XMPP. While STARTTLS is not formally specified for IRC, the InspIRCd implementation ${ }^{6}$ is generally considered a reference.

STARTTLS has the advantage that no dedicated port is required and that the communication partners can decide dynamically whether they want to use SSL/TLS. A major limitation is the vulnerability to active MitM attacks, where an attacker interferes with the STARTTLS-related commands. Unless clients or servers are specifically configured not to allow any connection without upgrade, the attack succeeds and the entire communication will be in plain. Depending on the user agent, users may not even be prompted with a security warning. The attack has been observed in the wild [9].

The SSL/TLS handshake is the same for both forms of connection establishment. The initiator sends an initial message together with information which symmetric cipher suites and SSL/TLS protocol versions it can support. The responder picks a cipher suite and negotiates a protocol version in its reply. It also sends an X.509 PKI certificate to authenticate. In another round trip, the cryptographic parameters-which may also include Diffie-Hellman parameters for forward secrecy-are then confirmed. The entities that wish to authenticate also include a proof that they are in possession of the private key that corresponds to their certificate.

It should be noted that email transfer over SSL/TLS is generally designed to prioritise successful transfer over any security guarantees. An opportunistic approach to security is often favoured by implementations: both initiator and responder may choose to ignore any authentication problems and proceed with message delivery despite errors or warnings.

\section{X.509 PKI}

In order for an entity to have trust into the authentication step, a number of conditions must be fulfilled that pertain to the configuration of the X.509 PKI in use. First and foremost, CAs must only issue certificates after applying due diligence in identifying the party that wishes to be certified. The CA/Browser forum has established guidelines for the Web use case [3]. The so-called Baseline Requirements define due diligence to require at least an (usually automated) check if the requesting party can receive email under the requested domain name and a specific email address. ${ }^{7}$ However, previous work has revealed cases where even this basic diligence was neglected. These cases are documented in, e.g., [23, 38]. On several occasions, CAs have been compromised. Since any CA may issue certificates for any domain, compromise of one CA is enough to compromise the entire PKI. More details on relevant

\footnotetext{
${ }^{6}$ https://wiki.inspircd.org/STARTTLS_Documentation

${ }^{7}$ There are alternatives, e.g., some form of token can be published on the web server, and some CAs apply further checks, e.g., lookups of WHOIS.
} 


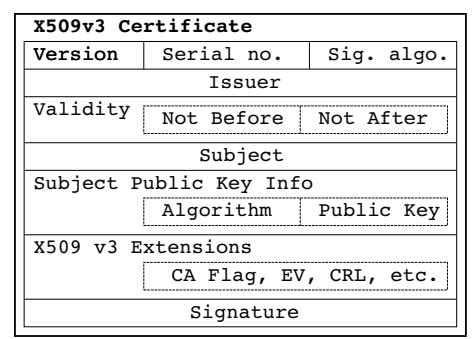

Fig. 1: An X.509 certificate.

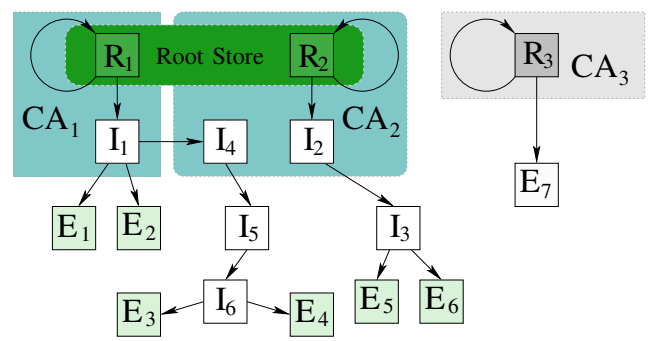

Fig. 2: X.509 PKI showing the most relevant features: root certificates, intermediate certificates, end-host certificates, a root store and a certificate signed by an untrusted CA.

attacks on the X.509 PKI for the Web can also be found in $[23,38]$. Notably however, X.509's use in email and chat protocols remains largely unexplored.

Fig. 1 shows the format of an X.509 certificate, and Fig. 2 shows a simplified PKI. The certificates of the CAs form trust anchors, which are distributed with operating systems and user agents like email clients (e.g., Thunderbird) or web browsers. For instance, the Windows and OS X operating systems come with root stores supplied by the vendors, who decide which CAs they include. Software on this OSes generally uses the OS-supplied trust anchors. Mozilla takes a different approach: their products have their own root store.

CAs can issue certificates directly (a practice that is thoroughly discouraged; see Section IV-F) or via intermediate certificates. Trust chains must not be broken, i.e., missing intermediate certificates, chaining to root certificates that are not in the root store, having expired certificates in the chain etc. Self-signed certificates, where root and end-host certificates are the same, are a special case, which we discuss in Section IV-D. Later in this paper, in Section IV, we will discuss problematic PKI setups after going through several observations from our measurements.

\section{Client-authentication methods}

The client-to-server communication protocols examined in this paper generally authenticate the initiator of the communication on the application layer and not in-band during the SSL/TLS handshake. SMTP did originally not require authentication for message submission (i.e., user agent to mail server), but this was added later to fight spam. Message transfer between MTAs (i.e., transfer from the source MTA to the destination MTA) does not require authentication of the sender.

To choose the appropriate authentication mechanism, a client is supposed to query the server for the mechanisms it supports (e.g., using the EHLO command with SMTP, or CAPABILITY for IMAP). The server returns a list of supported authentication mechanisms, sorted by preference, from which the client then selects.

Some of the most widely used mechanisms, LOGIN and PLAIN [46], transmit user credentials without further protection (independently of whether there is an underlying SSL/TLS connection or not). Some other mechanisms use cryptographic functions to transmit a hashed version of the credentials (often using deprecated hash functions such as MD5). An adversary who is able to eavesdrop on the authentication process can potentially recover the credentials. Challenge-response mechanisms such as CRAM [28] and SCRAM [33] (which also use HMAC) provide much better protection. With these mechanisms, the password is never transmitted at all. In the case of SCRAM, the password can even be stored in a salted format on server-side, and hence not even a server compromise would reveal the true password to an attacker.

\section{E. Related work}

A number of publications have studied the deployment of network security protocols, with a focus on either the development of generic, large-scale measurement methodologies or the measurement and analysis of the HTTPS and SSH protocols.

Provos and Honeyman [37] were probably the first to carry out academic, large-scale scans of security protocols. Their work focused on SSH. Later, Heidemann et al. carried out a census of Internet hosts [18]. Leonard and Loguinov [29] presented a scanner capable of carrying out Internet-wide scans with proper randomisation of target IP addresses. Durumeric et al. presented the fast zmap scanner in 2013 [6]. We used zmap in our work.

Vratonjic et al. [45] carried out a scan of the top 1 million hosts as determined by Alexa Inc. Holz et al. [24] carried out scans of the HTTPS ecosystem in a large-scale, long-term study over the duration of 18 months. The authors also used data from passive monitoring (using the Bro Network Monitor). The study showed the poor state of the Web PKI and predicted very little movement towards improvement. More recently, Durumeric et al. [7] presented an Internet-wide study of the HTTPS certificate ecosystem; Huang et al. [25] expanded on this in their investigation of forward-secure cipher deployments in TLS. Amann et al. [2] and Akhawe et al. [1] carried out two studies that analysed the aspects of trust relationships of the Web PKI and the occurrence and treatment of error cases during certificate validation in popular implementations, again using data from passive monitoring with Bro.

Some studies focused more on vulnerabilities in the wild. Heninger et al. [19] studied data sets won with zmap to investigate the cause and distribution of weak RSA and DSA keys. In their study of the Heartbleed vulnerability, Durumeric et al. [10] also found email and XMPP servers to be vulnerable. Gasser et al. [14] presented a large-scale study of the deployment of SSH in 2014, with a focus on the distribution of insecurely configured devices.

There are not many studies that would focus on the use of SSL/TLS beyond HTTPS. Concerning email, a recent study [9] 
actively probed the most popular email servers and observed the security of SMTP servers interacting with Gmail over the duration of a year. The authors found that the most popular providers did a decent job in setting up secure servers. A paper that was not yet published at the time of our initial submission also investigated the security of email server setups [12]. The authors limited themselves to a relatively small number of servers. However, an important finding of theirs is that SMTP servers often do not verify the correctness of a certificate in outgoing connections. In our own study, we extend our analysis to the whole Internet, but also to client-facing email retrieval protocols and chat protocols. On a global scale, our findings are not as reassuring as those for the most popular providers.

Finally, a number of online dashboards give some insight into the current deployment of SSL/TLS: SSL Pulse for the most popular websites ${ }^{8}$, Gmail about their SMTP peers ${ }^{9}$, or the IM observatory for XMPP servers ${ }^{10}$. The ICSI Certificate Notary $^{11}$ also offers an online, DNS-based query system that allows to check the validity of a given X.509 certificate.

\section{DATA COLLECTION}

We collected data using both active scans and passive measurement, i.e., traffic monitoring. We use our scans to characterise global TLS deployment. The use of passive monitoring data allows us to understand which specifics of TLS are actually used; e.g., which protocol versions and cipher suites are negotiated between communication partners. Active scans are not as suitable for this purpose: the responder chooses the cipher suite from the initiator's offers.

For email, we include all three SSL/TLS-variants of SMTP: SMTP with STARTTLS on port 25, SMTPS on port 465, and SUBMISSION with STARTTLS on port 587. For IMAP and POP3, we chose both the pure SSL/TLS as well as the STARTTLS variant. For XMPP, we investigate both client-toserver and server-to-server setups, in both STARTTLS and pure SSL/TLS variants. For IRC, we only investigate the client-toserver communication ${ }^{12}$. We limit our IRCS scan to the most common port, 6697, and probe for IRC STARTTLS support on the default IRC port, 6667.

\section{A. Active scans}

In this section, we describe the process we used to perform our active scans. We also explain some insights we gained and some peculiar phenomena we encountered when scanning.

a) Scanner: Our scanner consists of two parts. The first is the zmap [6] network scanner, which we used to determine IP addresses that had ports of interest open. We scanned the entire routable IPv4 space $^{13}$, using a BGP dump from the Oregon collector of Routeviews ${ }^{14}$ as a whitelist of routable prefixes. We ran our scanning campaigns over several weeks, from 2015-06-09 through 2015-08-04. Due to time-sharing constraints on the scanning machine, we had to run the scans

\footnotetext{
${ }^{8}$ https://www.trustworthyinternet.org/ssl-pulse/

${ }^{9} \mathrm{https}: / / \mathrm{www}$. google.com/transparencyreport/saferemail/

${ }^{10}$ https://xmpp.net/reports.php

${ }^{11} \mathrm{http}: / /$ notary.icsi.berkeley.edu/

${ }^{12}$ Recall that server-to-server communications are not standardised

${ }^{13}$ Appropriate ways to scan IPv6 are an open research topic.

${ }^{14}$ http://www.routeviews.org
}

at different speeds, resulting in scans of different durations, as summarised in Table I. In general, scans lasted roughly 20-36 hours. We refrained from scanning at line speed (although this is possible with our setup) to reduce our scans' impact.

The second part of our scanner is a component that starts an array of OpenSSL client instances, collects their output, and stores it in a database. We patched the STARTTLS implementations of OpenSSL as the current version does not follow the RFCs. More specifically, the current OpenSSL client does not query the server capabilities and ignores a server's refusal to negotiate SSL/TLS. Furthermore, OpenSSL did not yet support STARTTLS for IRC, either.

We used a blacklist of IP ranges generated during past scans $[14,42]$. At the time of writing, it contains 177 entries covering 2.6 million addresses (about $0.08 \%$ of the routable space). Entries were computed from both automated and personal emails that reached us and complained about the scans.

b) Scanned protocols: Table I gives an overview of our dataset from active scans. It shows the number of hosts responding to connection attempts as well as the number of hosts to which a successful SSL/TLS connection could be established. The table also lists the number of unique endhost certificates that we encountered on all machines in the respective scans. Furthermore, it contains the number of total and unique intermediate certificates encountered in the scans. Note that many servers seem to have a SSL/TLS port open, yet do not carry out successful SSL/TLS handshakes. This phenomenon has been observed before for HTTPS [6, 24]; we encounter it again for email and chat.

Previous scans performed by us show that servers that support only SSL 3 are very rare today. Modern Debian-based systems do not even include it in the default OpenSSL binary they ship. Initially, we followed their lead and did not try to connect with an optional fall-back to SSL 3. However, we revised that decision after inspecting data from the passive monitoring and deciding we wanted to allow for some comparisons. We thus enabled fall-back to SSL 3 for the remainder of our scans.

c) Background noise: We observed a phenomenon which has also been mentioned before by the zmap community: independently of the port one chooses to scan Internet-wide, there is always a number of hosts that reply to SYN packets without carrying out a full TCP handshake later. We verified this by scanning five arbitrarily chosen ports (1337, 7583, $46721,58976,65322$ ) and sending out 100M probes each time. We scanned twice with different seeds for each port. Every time, the average response rate was $0.07-0.1 \%$. When scanning protocols with very low deployment, it is important to keep this phenomenon in mind as one of the causes for failed SSL/TLS handshakes. This is particularly important to consider for less-used protocols such as IRC or XMPP.

\section{B. Passive collection}

For our passive measurements, we examined nine days of traffic of the Internet uplink of the University of California at Berkeley, which has a $10 \mathrm{GE}$ uplink with a peak traffic of more than $7 \mathrm{~GB} / \mathrm{s}$ each way. 
TABLE I: Description of our active scan dataset containing hosts listening on ports, successful handshakes, end-host and intermediate certificates. Entries marked with $\dagger$ used STARTTLS, and those with $\ddagger$ allowed fallback to SSL 3. S2S is short for server-to-server, C2S for client-to-server.

\begin{tabular}{|c|c|c|c|c|c|c|}
\hline Protocol & Port & Period & No. hosts & Successful SSL/TLS & Unique end-host-certs & Intermediate certs (unique) \\
\hline $\mathrm{SMTP}^{\dagger, \ddagger}$ & 25 & $7 / 27-7 / 28$ & $12,488,000$ & $3,848,843(30.82 \%)$ & $1,373,751(35.69 \%)$ & $2,243,846(23,462,1.05 \%)$ \\
\hline SMTPS $^{\ddagger}$ & 465 & $7 / 22-7 / 23$ & $7,234,817$ & $3,437,382(47.51 \%)$ & $800,574(23.29 \%)$ & $2,583,786(10,357,0.4 \%)$ \\
\hline $\operatorname{IMAP}^{\dagger, \ddagger}$ & 143 & $7 / 25-7 / 26$ & $8,006,617$ & $4,076,809(50.91 \%)$ & $1,024,757(25.14 \%)$ & $2,406,987(12,913,0.54 \%)$ \\
\hline IMAPS & 993 & $7 / 09-7 / 11$ & $6,297,805$ & $4,121,108(65.43 \%)$ & $1,053,110(25.55 \%)$ & $2,791,451(16,700,0.6 \%)$ \\
\hline $\mathrm{POP}^{\dagger, \ddagger}$ & 110 & $7 / 26$ & $8,930,688$ & $4,074,211(45.62 \%)$ & $998,013(24.5 \%)$ & $2,325,032(10,135,0.44 \%)$ \\
\hline IRCS & 6697 & $7 / 17-7 / 18$ & $1,948,656$ & $8661(0.44 \%)$ & $6332(73.11 \%)$ & $2551(315,12.35 \%)$ \\
\hline $\mathrm{XMPP}, \mathrm{C} 2 \mathrm{~S}^{\dagger, \ddagger}$ & 5222 & $7 / 29-7 / 30$ & $2,188,813$ & $53,544(2.44 \%)$ & $38,916(63.61 \%)$ & $5927(1913,32.28 \%)$ \\
\hline XMPPS, C2S & 5223 & $7 / 13-7 / 14$ & $2,223,994$ & $70,441(3.16 \%)$ & $38,916(55.25 \%)$ & $32,629(2773,8.5 \%)$ \\
\hline XMPP, S2 $\mathrm{S}^{\dagger, \ddagger}$ & 5269 & $7 / 31-8 / 01$ & $2,459,666$ & $9780(0.39 \%)$ & $6221(63.61 \%)$ & $5927(1913,32.28 \%)$ \\
\hline XMPPS, S2S $\ddagger$ & 5270 & $7 / 24$ & $2,046,204$ & $1693(0.08 \%)$ & $1146(67.69 \%)$ & $783(147,18.77 \%)$ \\
\hline
\end{tabular}

TABLE II: Connections and servers in passive scans. Entries marked with $\dagger$ used STARTTLS. S2S is short for serverto-server, C2S for client-to-server.

\begin{tabular}{lrrr}
\hline \multicolumn{1}{c}{ Protocol } & Port & Connections & Servers \\
\hline SMTP $^{\dagger}$ & 25 & $3,870,542$ & 8626 \\
SMTPS & 465 & 37,306 & 266 \\
SUBMISSION $^{\dagger}$ & 587 & $7,849,434$ & 373 \\
IMAP $^{\dagger}$ & 143 & 25,900 & 239 \\
IMAPS & 993 & $4,620,043$ & 1196 \\
POP3 $^{\dagger}$ & 110 & 18,774 & 110 \\
POP3S & 995 & 159,702 & 341 \\
\hline IRC $^{\dagger}$ & 6667 & 53 & 2 \\
IRCS $_{\text {XMPP, C2S }}^{\dagger}$ & 6697 & 18,238 & 15 \\
XMPPS, C2S $_{\text {XMPP, S2S }}^{\dagger}$ & 5222 & 13,517 & 229 \\
XMPPS, S2S $^{\dagger}$ & 5269 & 911,411 & 2163 \\
\hline
\end{tabular}

a) Traffic monitoring and capture: We used the Bro Network Security Monitor ${ }^{15}$ [35] to gather information about all outgoing SSL/TLS sessions. In a default installation, Bro already offers deep visibility into standard SSL/TLS traffic, extracting certificates and meta-information like cipher and key use. For this work, we extended Bro to also work with protocols using STARTTLS. We added support for STARTTLS for the SMTP, POP3, IRC, XMPP, and IMAP protocols.

We also use Bro to extract the server's offered authentication capabilities for all outgoing SMTP, POP3, and IMAP sessions, which allows us to deduce how many of the contacted servers support STARTTLS. We added support for capabilities to the IMAP protocol analyser we created for this work; support was already present in Bro for SMTP and POP3 capabilities.

Our passive dataset was collected from 2015-07-29 to 2015-08-06. We observed a total of 9,730,095 SSL/TLS connections on the monitored ports. The connections were established

\footnotetext{
${ }^{15}$ http://www.bro.org
}

to 12,637 unique destination IP addresses with 10,294 distinct Server Name Indication (SNI) values and 10,164 unique endhost certificates. Table II shows the number of connections and servers encountered per port.

Please note that our passive data set exhibits artefacts of the collection process that are beyond our control. As our data is collected at the Internet uplink of one university, it is potentially biased. We assume that, due to the high number of students with diverse cultural backgrounds, the traffic we see is similar to traffic in other parts of the world, however.

b) Ethical considerations: We are aware of the ethical considerations that must be taken into account when observing passive traffic. This research strives to understand the interplay between server and client software at the technical level and does not concern any human subjects. For the SSL/TLS measurements, the information that we save is constrained to information in the SSL/TLS handshake without analysing any later connection payload data. The campus administration has approved this data collection. For the capability measurements, only automatic server capability replies were recorded, which do not contain any personally identifiable information. In addition, the University IRB takes the position that IP addresses, which were also recorded for this measurement, are not treated as personally identifiable.

c) Unusual traffic on standard ports: While analysing the TLS extensions sent by clients, we noticed that there are 4,584 connections that send the Application-Layer Protocol Negotiation (ALPN) extension, which is used to negotiate protocols like HTTP2 and SPDY. Closer examination shows that 2,703 of these connections going to six servers indeed contain values that point to them being HTTPS servers, running on port 993 as well as 110. Manually connecting to a few of these IP addresses shows that they are Squid proxy servers running on non-standard ports. The remaining 1,881 connections to 780 hosts all have a destination port of 5223. The ALPN in these cases indicates a value of apns-security-v1 and apns-security-v2, terminating at nodes for the Apple push notification service. We are not sure what software causes 
TABLE III: STARTTLS support and use. Passive monitoring allows to differentiate server-side support from client-server connections which were actually negotiated. S2S is short for server-to-server, C2S for client-to-server.

\begin{tabular}{lrrrr}
\hline & Active probing & \multicolumn{3}{c}{ Passive monitoring } \\
\cline { 2 - 5 } \multicolumn{1}{c}{ Protocol } & $\begin{array}{c}\text { Supported } \\
\text { \& upgraded }\end{array}$ & $\begin{array}{c}\text { Supporting } \\
\text { servers }\end{array}$ & $\begin{array}{c}\text { Offering } \\
\text { connections }\end{array}$ & $\begin{array}{c}\text { Upgraded } \\
\text { connections }\end{array}$ \\
\hline SMTP & $30.82 \%$ & $59 \%$ & $97 \%$ & $94 \%$ \\
SUBMISSION & $43.03 \%$ & $98 \%$ & $99.9 \%$ & $97 \%$ \\
IMAP & $50.91 \%$ & $77 \%$ & $70 \%$ & $44 \%$ \\
POP3 & $45.62 \%$ & $55 \%$ & $73 \%$ & $62 \%$ \\
\hline IRC & $0.14 \%$ & - & - & - \\
XMPP, C2S & $2.44 \%$ & - & - & - \\
XMPP, S2S & $0.39 \%$ & - & - & - \\
\hline
\end{tabular}

these connections. Further traffic analysis also reveals that our data set contains 3,728 certificates, from 9,082 connections to 110 servers, indicating that they are used by the Tor service. We excluded all these servers from further analyses.

d) OCSP stapling: Another interesting finding is the adoption of OCSP [31] stapling by email servers. OCSP stapling allows TLS servers to send a proof that their certificate is currently still valid and has not been revoked. This is part of the TLS handshake if the client signals support for the extension. We encountered 836 connections using OCSP stapling, terminating at 64 different servers. The majority of these (706 connections and 58 servers) were on port 993 (IMAPS).

\section{SECURITY ANALYSIS}

We now analyse our datasets from a security perspective. Specifically, we examine how appropriately servers are configured. We look at basic parameters such as the ciphers in use and also consider PKI-related specifics, such as whether the offered certificates are valid and linkable to CAs present in the Mozilla root store, the amount of key and certificate reuse, and whether CAs follow best practices in issuing certificates. We finally study authentication methods offered to clients. Where applicable, we compare our findings for email and chat protocols with results from our HTTPS scan.

\section{A. Use of STARTTLS vs. direct SSL/TLS}

As mentioned in Section II, email and chat protocols can be secured with SSL/TLS either by using SSL/TLS on a dedicated port or by upgrading a TCP connection via STARTTLS.

Table I shows how many hosts supported SSL/TLS directly. We also measured support for STARTTLS in our active and passive scans (see table III). Our data shows that, depending on the application-layer protocol, about 30 to $51 \%$ servers offer STARTTLS. The STARTTLS extension is also often used in practice. While popular servers seem to support the extension (and thus most connections contain an offer to use it), the results for SMTP, IMAP, and POP3 do show that there is a significant number of servers without support. At least in the case of IMAP and POP3, one can also see that, in a considerable number of cases, connections are not upgraded although the server would support it.
TABLE IV: Negotiated protocol versions from active scans with SSL 3 activated and passive monitoring.

\begin{tabular}{lrr}
\hline Version & $\begin{array}{c}\text { Active probing } \\
\text { Negotiated with server }\end{array}$ & $\begin{array}{c}\text { Passive monitoring } \\
\text { Observed connections }\end{array}$ \\
\hline SSL 3 & $0.02 \%$ & $1.74 \%$ \\
TLS 1.0 & $39.26 \%$ & $58.79 \%$ \\
TLS 1.1 & $0.23 \%$ & $0.1 \%$ \\
TLS 1.2 & $60.48 \%$ & $39.37 \%$ \\
\hline
\end{tabular}

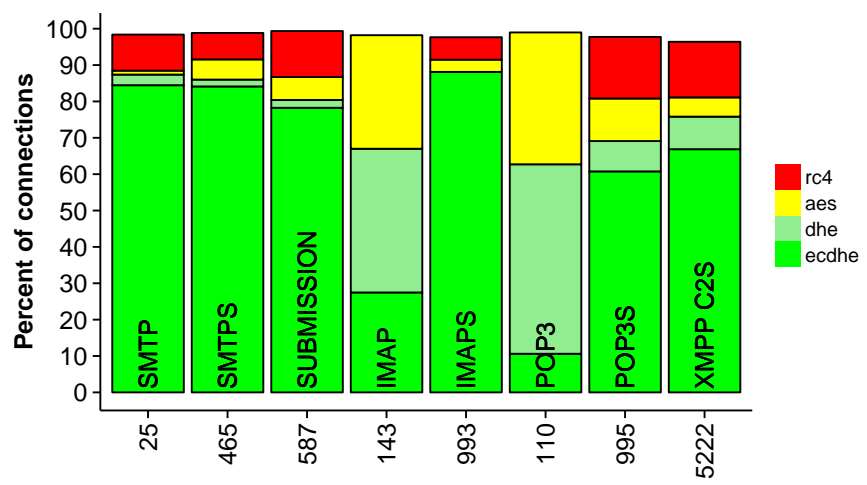

Fig. 3: Use of PFS ciphers by port. Red and yellow indicate that PFS is not used.

\section{B. SSL/TLS versions-deployment and use}

Ideally, only the latest version of TLS (1.2) should be used. Previous versions, especially SSL 3, have vulnerabilities, many of which are listed in RFC 7457 [44].

TABLE IV shows how often the different SSL/TLS protocol versions were chosen by servers in active scans (in scans that supported SSL 3). Note that we did not scan for SSL 2. TABLE IV also shows protocol versions observed in use in our passive monitoring dataset. No connections using SSL 2 were encountered.

Our result shows that just $0.03 \%$ of scanned servers only support the old and relatively insecure SSL 3-all others preferred one of the stronger TLS versions. However, the percentage of connections actually using SSL 3 in our passive dataset is much higher $(1.74 \%)$. There are two possible reasons for this. Either clients connect preferentially to less secure servers-this would not be in line with our results for STARTTLS support in the previous section. Or there is a significant number of clients that offer SSL 3 only, e.g., because they are outdated.

\section{Cipher use}

In SSL/TLS, the server chooses the symmetric cipher to use, based on a list of ciphers that the client suggests. Determining which ciphers a server supports would require many connections to test all ciphers individually. Given that many of those suites may never be negotiated, this is a poor trade-off in terms of good Internet citizenship versus lessons that can be learned.

We thus use passively monitored data to investigate which ciphers are actually negotiated in practice. Due to the high 


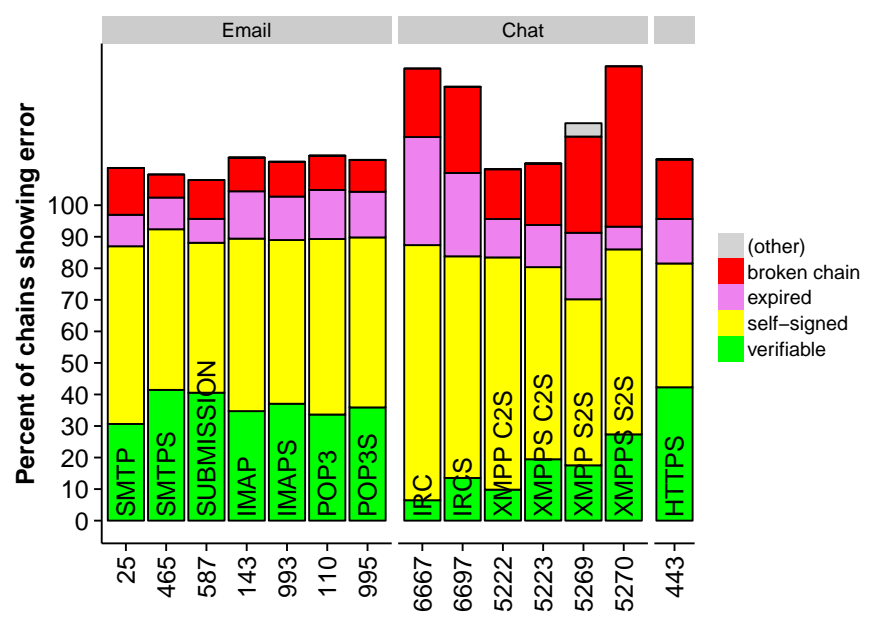

Fig. 4: Common errors in certificate chains, active scans. Note that chains may exhibit more than one error, which we capture in this figure. Thus, the results may add up to more than $100 \%$.

number of different cipher suites occurring in the wild- 35 in our dataset-we group the ciphers into categories that show their relative strengths. Figure 3 shows the different categories. The categories ECDHE and DHE contain suites that use forward-secure (PFS) ciphers, either using elliptic curve or modular Diffie-Hellmann key exchanges. The categories AES and RC4 contain connections without PFS support that use either the AES or RC4 cipher. Other categories with a use of less than $1 \%$ of connections were omitted (an example for this are connections using the Camellia cipher). Connections on ports 6679 and 6667 overwhelmingly use ECDHE ciphers, and those on port 5269 overwhelmingly use DHE ciphers. These ports were excluded from the figure for brevity. Figure 3 shows that there is still a surprisingly large amount of connections on some ports that use the RC4 stream cipher.

Looking at the elliptic curves that are used in ECDHE key exchanges reveals that $97.2 \%$ of connections use the secp $256 r 1$ curve, followed by $2 \%$ using secp $384 r 1$ and $0.78 \%$ using sect $571 r 1$. All of these curves are considered to be at least as strong as 2048 bit RSA, raising no immediate security concerns. This result is similar to earlier results concerning server support for different curves [25].

Examining the Diffie-Hellmann parameter sizes for the DHE connections reveals that $76 \%$ of the connections use a parameter size of 1024 bit, $22 \%$ of 2048 bit, and $1.4 \%$ of 768 bit. While this is an improvement in comparison to earlier studies, which measured more than $99 \%$ of hosts only supporting 1024 bit keys and below (see [25]), this is still relatively poor as parameter sizes below 2048 bits are discouraged today.

\section{Certificate chain validity}

SSL/TLS servers send a certificate chain in the handshake that consists of the host's certificate and potentially intermediate and CA certificates. It is common to omit the CA certificate as it already has to be part of the local root store. Chains can exhibit several types of errors-certificates may be expired, host certificates may not chain up to a root certificate in the root

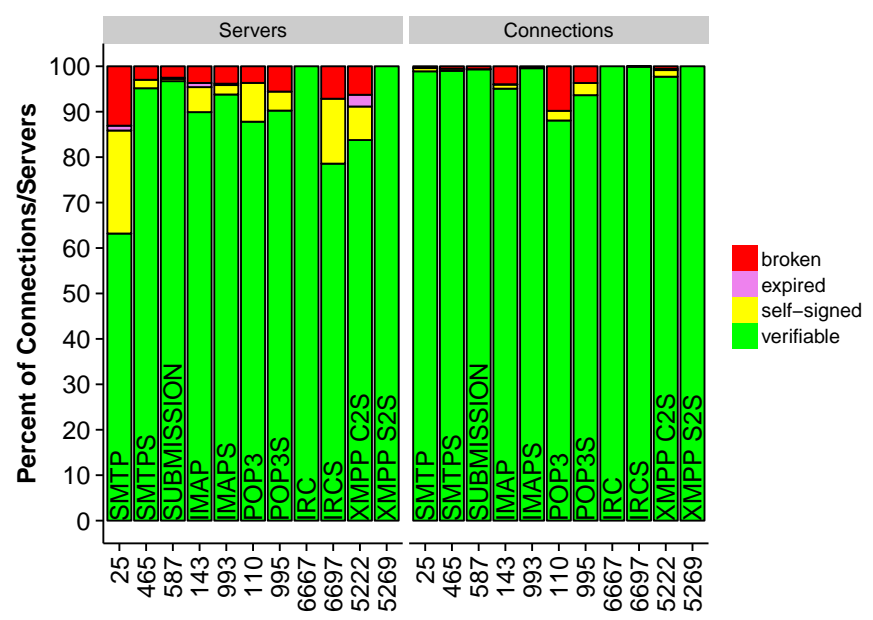

Fig. 5: Common errors in certificate chains, passive scans. Only the primary error (as reported by OpenSSL) is shown.

store, intermediate certificates can be missing, etc. A particularly common case are self-signed certificates, where issuer and subject of the certificate are the same. While technically not an error, these certificates can only serve the use case where a private server operator does either not care about authenticated encryption (and thus often uses some standard certificate as supplied in, e.g., Linux distributions) or issues a certificate to herself and configures her clients to accept it. ${ }^{16}$

a) Deployed vs. used services: We show the most common certificate errors we encountered in our active scans in Fig. 4. Fig. 5 shows validation results for our passive monitoring run by servers (i.e., counting every server once) and weighted by connections (i.e., counting each server weighted by the amount of connections that we saw).

This data set contains 295 cases where the same IP and port serves more than one certificate chain. Examples for this are Google and other company mail servers, servers where only CA certificates were updated while the end-host certificate was left unchanged, and servers renewing their end-host certificates. We examined the certificates sent by these servers and found that they all share the same validity characteristics (i.e., in all cases either all of the certificates sent by a host were valid or invalid).

Fig. 4 shows that the ratio of verifiable chains is between $30-40 \%$ across all email protocols. This is much lower than what has been reported for web sites on the Alexa Top 1 million list (around 60\%) [24], but much more in line with what has been found for the Web PKI as a whole [7]. For comparison, we also included the values for HTTPS. Looking at the data from passive monitoring, we note that the number of correctly validating chains is much higher when only considering servers that actually receive connections, and even more so when weighting this with the number of connections, as shown in Fig. 5. This suggests that the operators of the most popular

\footnotetext{
${ }^{16}$ Many clients allow to do this by storing an exception for the host and certificate on the first connection, thus making all subsequent connections secure as the stored certificate is compared against the one the server sends.
} 
services do a substantially better job at properly configuring their server for use with SSL/TLS.

b) Invalid certificates: Self-signed certificates are the major source of non-verifiable certificate chains in our measurements. As mentioned above, clients that wish to authenticate servers configured with such certificates must have out-of-band knowledge about the correctness of the certificate. Note that this approach only works where a self-signed certificate was created by the administrator-default certificates, as they are often shipped with software bundles, are useless for authentication as a copy of the private key is also shipped with the bundle. This is one case for certificate reuse, which we discuss below.

Certificate chains can be broken in a number of wayse.g., missing intermediate certificates, using CA certificates that are not in the root store, etc. We grouped these errors together and found that their number is relatively low at 10$15 \%$. Our result shows that, just as in the Web PKI, there are many mistakes that can be made in certificate deployment. The number of expired certificates, which we consider separately, is well within previously reported ranges [7, 24], showing that there is little difference between email and web protocols in this regard. We also found some further errors in certificate chains that we classified as 'other'-these are rare and sometimes somewhat arcane ${ }^{17}$. Just as in previous scans [24], we found only a single-digit number of cases with broken signatures.

Looking at the different protocols in Fig. 4, we see a difference between the email protocols and the chat protocols. While SMTPS and SUBMISSION have the highest (yet still unsatisfactory) percentage of verifiable certificate chains (and IMAP, POP3, and SMTP are trailing not too far behind), the numbers are much lower for XMPP and especially IRC. SMTP also has a much lower rate of verifiable certificate chains in our passive scans, at least when not weighting by number of connections: an indication that message protection in a number of server-to-server communications is likely to be at higher risk (although once again, popular servers seem to be properly configured). This is a serious problem, which is also compounded by the findings of a recent study that ran in parallel to ours [12]: the authors found that the servers in their study did not verify certificates in outgoing connections at all. It is thus reasonable to assume that many SMTP server-to-server connections are not secure.

A staggering number of IRC servers seems to use selfsigned certificates, or deploy broken or expired chains. This puts private (person-to-person) IRC messaging as well as password transfers at risk. We study the case of XMPP separately below.

c) The case of XMPP: The vast majority of certificates deployed for the XMPP client-to-server services (5222 and 5223) are self-signed. However, an inspection of typical common names for these certificates shows that the corresponding servers are most likely parts of proprietary deployments and not intended for general use. The corresponding subjects for XMPP on port 5222 are shown in TABLE VI. For XMPPS on port 5223, $48 \%$ were from a Content Distribution Network (incapsula.com), 12\% from Apple's push service (courier.push.apple.com) and another $8 \%$ by a Samsung push service (*.push.samsungosp.com).

\footnotetext{
${ }^{17}$ A full list of possible errors can be found on the OpenSSL homepage; https://www.openssl.org/docs/apps/verify.html.
}

The remaining certificates have shares between 2 and $5 \%$ and contain variations of the subjects hub. clickmyheart. net, i cewarp. com and ejabberd-a popular XMPP implementation. We thus conclude that this port is often used for push services, rather than instant messaging.

Consulting our passive data set confirms this conclusion. $90 \%(826,822)$ of port 5223 connections to 1,282 servers use a SNI containing push.apple.com, with all but two of the server IP addresses residing in Apple's IP space ${ }^{18} .73,465$ more connections target the Samsung service mentioned above, pushing the connection numbers to these services beyond $99 \%$ of all port 5223 connections. Our passive observations also show that the majority of client-to-server connections have verifiable chains. This is also true when looking at the distribution for servers only, albeit to a lesser degree. Once again we see a preferential use of servers with better-than-common security.

For the server-to-server ports, which are used to relay XMPP messages, we found broken (and not self-signed chains) to be slightly more common in our active scans (but notably not in our passive data). It is difficult to arrive at a strong conclusion here. The slightly lower percentage of self-signed certificates may hint at conscious certification choices made for server-to-server communication. Since XMPP is also used in proprietary products (not meant for public access), operators may have chosen to use private CAs instead of acquiring certificates from commercial CAs. If true, we did not capture such communication in our passive observations.

\section{E. Key and certificate reuse}

a) Certificate reuse: Holz et al. [24] showed that certificates are often reused on different IP addresses. Although IP addresses do not equal actual hosts, the frequency at which this phenomenon occurred provided strong indications that reuse across machines was happening. We investigated this phenomenon here, too. One potential reason for certificate reuse are Content Distribution Networks (CNDs). This is a legitimate use case where the ease of key distribution has to be balanced against a slightly increased attack surface. One would expect a clear difference in the distributions for valid and invalid certificate chains in this case as CDNs can be assumed to exercise care in deployment. Another possibility are default certificates, potentially from software bundles or deployed by management tools, which are not changed when the server is further configured.

Fig. $6 \mathrm{a}$ and $6 \mathrm{~b}$ plot the likelihood that 'a certificate occurs on $X$ IPs' for the entire set of certificates and only for the set of certificates that have correct certificate chains, respectively. While the results for the Web PKI [24] revealed a clear difference between the subset of certificates with valid chains and the overall set of certificates, this is much less pronounced here. Furthermore, the likelihood is almost the same across the email protocols. The only real difference can be seen for XMPP and IRC - however, we need to stress the smaller number of verifiable certificate chains we have for these two protocols.

We also investigated the reuse of self-signed certificates. If these are created purposefully for a single server or service, they

\footnotetext{
${ }^{18}$ The remaining two addresses with one connection per address use an IPv6 target address in an address space a network provider uses for NAT64; we assume these addresses also get redirected to Apple servers in some way.
} 


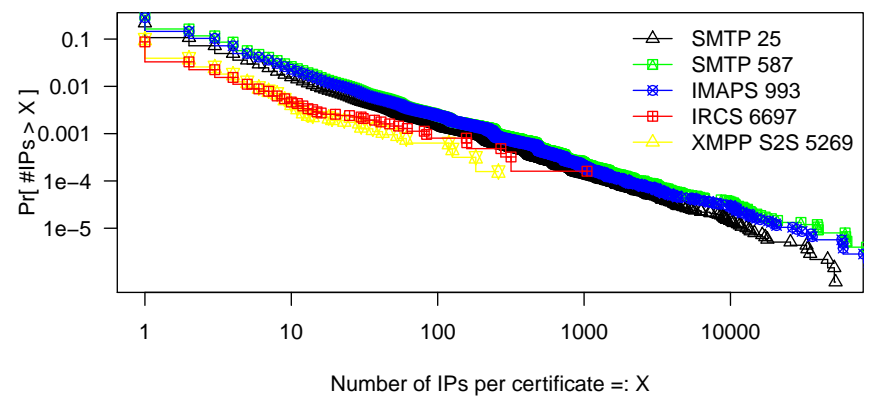

(a) All certificates

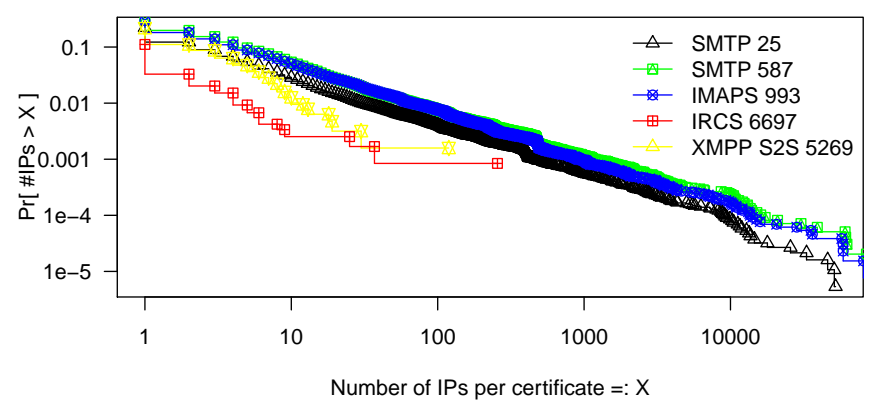

(b) Valid certificates only

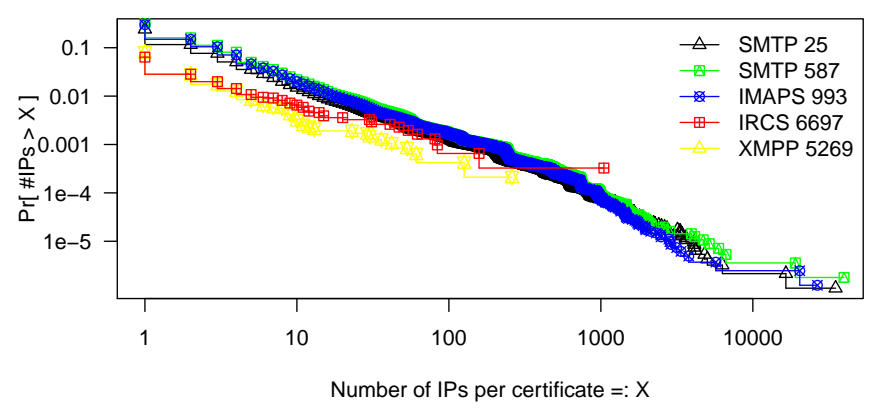

(c) Self-signed certificates only

Fig. 6: Likelihood that a certificate is used on $X$ IPs. SMTP 587 is SUBMISSION.

should not occur on too many hosts. Figure 6c shows, however, that many appear on hundreds or thousands of hosts. Hence, a more likely explanation is that these are default certificates shipped with software.

The reuse of certificates is naturally reflected in the number of public keys that are unique to a host, shown in Figure 7. Only about $15 \%$ of public keys occur on exactly one host.

b) Popularity of servers reusing cryptographic material: We investigated whether passive monitoring would yield similar

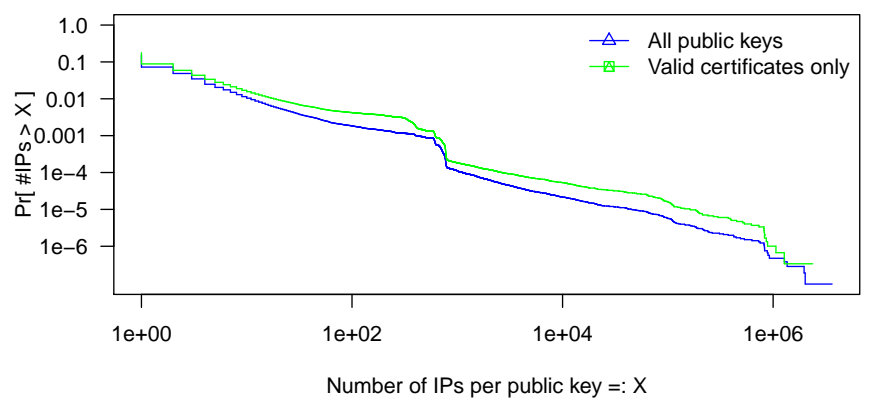

Fig. 7: Likelihood that a public key is used on $X$ IPs, across all hosts and certificates.

TABLE V: Duplicate certificates by port in passive scans. Entries marked with $\dagger$ used STARTTLS.

\begin{tabular}{lrrr}
\hline \multicolumn{1}{c}{ Protocol } & Port & Dup. Certs & Valid Dup. Certs \\
\hline SMTP $^{\dagger}$ & 25 & 877 & 656 \\
SMTPS & 465 & 36 & 36 \\
SUBMISSION $^{\dagger}$ & 587 & 46 & 46 \\
IMAP $^{\dagger}$ & 143 & 29 & 28 \\
IMAPS $^{\dagger}$ & 993 & 119 & 111 \\
POP3 $^{\dagger}$ & 110 & 12 & 12 \\
POP3S & 995 & 43 & 41 \\
\hline IRCS $_{\text {XMPP, C2S }}^{\dagger}$ & 6697 & 3 & 3 \\
\hline
\end{tabular}

results for key reuse. We expected a very different picture as we assume Internet users to mostly access services of larger providers, which are much more likely to use correctly deployed certificate chains.

In our passive monitoring run, 1,096 (17\%) of our 6,398 encountered certificates were seen on more than one IP address. Table V shows the prevalence of certificate reuses per port. As the table shows, the majority of certificate reuses happens on port 25 .

Furthermore, in our passive scans $78 \%$ of all certificates that we see on at least 2 hosts are valid, hinting towards the fact that many hosting providers use this for load balancing. Indeed, examining the certificates that were seen on the most IP addresses show a SMTP certificate by Proofpoint, Inc. that was encountered on 263 IPs, followed by Google certificates for imap.gmail.com (184) and mx.google.com (161).

This shows that, while there is a rampant amount of certificate reuse on the Internet as a whole, many of these servers seem not to be contacted commonly by clients, hinting at a considerable server population that might be for private use or only used by a small user population.

c) Common names: We show the Common Names in some particularly common and invalid certificates in TABLE VI.

Note that we cannot study if the subjects in certificates match the host names where the certificates are deployed. This would require scans based on a target list of domain names 
TABLE VI: Common names in particularly frequently occurring and invalid certificates for SMTP, IMAP, XMPP. $\dagger$ indicates data obtained during a STARTTLS negotiation.

\begin{tabular}{lr}
\hline \multicolumn{1}{c}{ Common name } & Occurrences \\
\hline \multicolumn{1}{c}{ SMTP } \\
\end{tabular}

and comparing the subjects in the received certificates with the expected domain name. However, we only scanned by IP addresses. Reverse DNS lookups could theoretically produce domain names to compare against; however, due to the way that servers are operated today, there is a risk that the reverse lookup yields hostname aliases different from the actual domain name by which the server is typically addressed.

Some interesting findings for SMTP on port 25 are as follows. The certificates for $\star$. bi zmw contain the string 'NTT Communications Corporation' in the 'Organisation' part of the subject, a hint in which organisation these invalid certificates are used. The certificates for 'localhost' that are marked with an asterisk all contain the string 'Qmail Toaster Server', thus indicating that the responsible SMTP server was the popular Qmail by djb. Presumably, the operators had never bothered to install proper certificates. The 'webaster' certificate had already made an appearance in the Web PKI study [24] and is most likely due to a certificate creation software with a spelling weakness. Plesk is the company behind the Parallels visualisation product.

For IMAPS, we also find the popular 'webaster' certificate. Furthermore we find certificates of several hosting companies and also of Hurricane Electric. The certificates for Microsoft were a surprise as they seemed to contain a Web address for the Windows Update service. There were 18,193 occurrences
TABLE VII: Invalid Microsoft certificates: ASes and CIRCL ranking for botnet and malicious activity.

\begin{tabular}{llr}
\hline AS number & \multicolumn{1}{c}{ Registration information } & CIRCL rank \\
\hline 3257 & TINET-BACKBONE Tinet SpA, DE & 9532 \\
3731 & AFNCA-ASN - AFNCA Inc., US & 4804 \\
4250 & ALENT-ASN-1 - Alentus Corporation, US & 9180 \\
4436 & AS-GTT-4436 - nLayer Communications, Inc., & 10,730 \\
& US & \\
6762 & SEABONE-NET TELECOM ITALIA & 11,887 \\
& SPARKLE S.p.A., IT & 557 \\
11346 & CIAS - Critical Issue Inc., US & 6255 \\
13030 & INIT7 Init7 (Switzerland) Ltd., CH & 4139 \\
14618 & Amazon.com Inc., US & 3143 \\
16509 & Amazon.com Inc., US & 4712 \\
18779 & EGIHOSTING - EGIHosting, US & 2828 \\
21321 & ARETI-AS Areti Internet Ltd.,GB & \\
23352 & SERVERCENTRAL - Server Central Net- & - \\
26642 & work, US & 6330 \\
41095 & AFAS - AnchorFree Inc., US & - \\
54500 & IPTP IPTP LTD, NL & \\
\hline
\end{tabular}

of this single end-host certificate. No intermediate or root certificates were sent. The respective hosts were distributed across 15 Autonomous Systems, which we looked up using the Team Cymru ASN Database ${ }^{19}$.

TABLE VII shows the results of the lookups. None of the ASes were registered to Microsoft; they were predominantly assigned to hosters. We checked the BGP ranking of these ASes with CIRCL's web site, which as of 12 August 2015 contained 12,339 ASes ranked for known botnet and malicious activity. Only two of the ASes were not on that list. Manual inspection of the certificate did not yield anything out of the ordinary, however. We contacted Microsoft repeatedly (directly and via CIRCL), but never received a response why such a certificate should occur on an email port.

Analyzing XMPP certificates also yielded some interesting results. OneX is an XMPP server by Avaya, a communications company-this seems to be a default certificate. Fonality is a provider of unified messaging. The certificates for $\mathrm{k} 66$. ru contained a string referring to a product called 'CommuniGate'. It also appeared in the certificates for visn. net. We were unable to determine the exact nature of clickmyheart, but the Web site shows a login portal, so we presume some forum. The certificates also contained the name Zimbra Collaboration server. 'John Doe' is used in the certificates by Jive Software. Java2go seems to be an SMS product.

Beyond the strange Microsoft Update certificate, our results suggest that a number of default keys and certificates are used in production. This is a negative finding as it means that other parties may have access to the private cryptographic material. Some vendors, meanwhile, seem to choose their own, private CA instead of working with a commercial one.

\section{F. Poor CA practice}

In our data set, we were still able to find certificates that were issued directly from a root CA without any intermediate certificate. The industry has moved away from this practice and discourages it today [3]. To issue such certificates, the CA's

\footnotetext{
${ }^{19}$ https://asn.cymru.com/cgi-bin/whois.cgi
} 
TABLE VIII: Authentication mechanisms offered by servers on SUBMISSION port.

\begin{tabular}{lrr}
\hline \multicolumn{1}{c}{ Mechanism } & Advertised & Servers \\
\hline PLAIN & $2,764,157$ & $99.27 \%$ \\
LOGIN & $2,760,100$ & $99.15 \%$ \\
CRAM-MD5 & 431,634 & $15.50 \%$ \\
DIGEST-MD5 & 230,152 & $8.26 \%$ \\
OTP & 19,850 & $0.71 \%$ \\
GSSAPI & 16,555 & $0.59 \%$ \\
NTLM & 13,663 & $0.49 \%$ \\
XOAUTH2 & 3118 & $0.11 \%$ \\
PLAIN-CLIENTTOKEN & 1642 & $0.05 \%$ \\
XOAUTH & 1641 & $0.05 \%$ \\
\hline Other 591 mechanisms found & 5329 & $0.19 \%$ \\
\hline
\end{tabular}

TABLE IX: Authentication mechanisms offered by servers on IMAPS port.

\begin{tabular}{lrr}
\hline \multicolumn{1}{c}{ Mechanism } & Advertised & Servers \\
\hline PLAIN & $3,753,658$ & $96.66 \%$ \\
LOGIN & $2,430,559$ & $62.59 \%$ \\
CRAM-MD5 & 467,460 & $12.04 \%$ \\
CRAM-SHA1 & 186,355 & $4.80 \%$ \\
CRAM-SHA256 & 185,427 & $4.77 \%$ \\
DIGEST-MD5 & 160,893 & $4.14 \%$ \\
GSSAPI & 18,851 & $0.49 \%$ \\
NTLM & 17,106 & $0.44 \%$ \\
X-ZIMBRA & 7582 & $0.20 \%$ \\
MSN & 4181 & $0.11 \%$ \\
\hline Other 61 mechanisms found & 6773 & $0.17 \%$ \\
\hline
\end{tabular}

root certificate needs to be kept online, a serious attack vector. A root certificate compromise would necessitate an update of all clients that include it. We expect the number of certificates that are directly issued from a root certificate to shrink further.

Indeed, there were very few cases already in our scan. For SMTP, for instance, we found only 794 cases, or $0.07 \%$ of verifiable chains. The percentages for SUBMISSION was $0.08 \%$. Interestingly, it was $0.5 \%$ for SMTPS. SMTPS is deprecated-it is not implausible that operators who still enable SMTPS have simply never upgraded to new, intermediate-issued certificates. For good measure, we also tested this property for the two IRC protocols (only one case for IRCS), the two XMPP client-to-server variants (two for STARTTLS, 14 for XMPPS), and the two XMPP server-to-server variants (one case each).

\section{G. Authentication methods}

We analysed the authentication mechanisms supported and advertised by servers to clients when sending mails using SUBMISSION or retrieving mails with IMAPS. In our active measurements, we queried the servers for authentication capabilities using the EHLO command for SUBMISSION and the CAPABILITIES command for IMAPS. Capabilities were always queried after TLS session establishment. We show the most common authentication mechanisms in Tables VIII and IX.

The results obtained for both SUBMISSION and IMAPS show poor support for strong authentication mechanisms. Mechanisms transmitting credentials in plaintext (PLAIN and LOGIN) are supported by more than $99 \%$ of the SUBMISSION
TABLE X: Combinations of authentication mechanisms offered by servers on SUBMISSION port.

\begin{tabular}{lrr}
\hline \multicolumn{1}{c}{ Mechanism } & Advertised & Servers \\
\hline PLAIN, LOGIN & $2,092,594$ & $75.15 \%$ \\
LOGIN, PLAIN & 224,197 & $8.51 \%$ \\
LOGIN, CRAM-MD5, PLAIN & 96,322 & $3.45 \%$ \\
LOGIN, PLAIN, CRAM-MD5 & 45,477 & $1.63 \%$ \\
DIGEST-MD5, CRAM-MD5, PLAIN, LOGIN & 36,416 & $1.30 \%$ \\
CRAM-MD5, PLAIN, LOGIN & 29,046 & $1.04 \%$ \\
PLAIN, LOGIN, CRAM-MD5 & 24,914 & $0.89 \%$ \\
CRAM-MD5, DIGEST-MD5, LOGIN, PLAIN & 19,877 & $0.71 \%$ \\
PLAIN & 17,079 & $0.61 \%$ \\
\hline Other 1234 combinations found & 326,392 & $7.11 \%$ \\
\hline
\end{tabular}

TABLE XI: Combinations of authentication mechanisms offered by servers on IMAPS port.

\begin{tabular}{lrrr}
\hline \multicolumn{1}{c}{ Mechanism } & Advertised & Servers \\
\hline PLAIN, LOGIN & & $2,222,721$ & $60.16 \%$ \\
PLAIN & 982,386 & $26.59 \%$ \\
CRAM-MD5, CRAM-SHA1, CRAM-SHA256, & 183,813 & $4.97 \%$ \\
PLAIN & & \\
CRAM-MD5, PLAIN & 90,341 & $2.45 \%$ \\
PLAIN, LOGIN, DIGEST-MD5, CRAM-MD5 & 78,061 & $2.11 \%$ \\
LOGIN & 21,842 & $0.59 \%$ \\
CRAM-MD5, PLAIN, LOGIN, DIGEST-MD5 & 16,660 & $0.45 \%$ \\
PLAIN, LOGIN, CRAM-MD5 & 10,731 & $0.29 \%$ \\
CRAM-MD5, PLAIN, LOGIN, DIGEST-MD5, & 9105 & $0.25 \%$ \\
NTLM & & 7569 & $0.20 \%$ \\
PLAIN, X-ZIMBRA & & 71,685 & $1.94 \%$ \\
\hline Other 1039 combinations found & & \\
\hline
\end{tabular}

and $90 \%$ of the IMAPS servers. On the other hand, less than $16 \%$ of the SUBMISSION and $12.04 \%$ of the IMAPS servers support much stronger mechanisms such as CRAM.

This is made worse by the fact that the vast majority of SUBMISSION $(84.86 \%)$ and IMAPS $(87.43 \%)$ servers support only PLAIN and LOGIN. The ordering of authentication mechanisms is not particularly encouraging, either: clients obeying the ordering suggested by many servers will use a plaintext mechanism for SUBMISSION (resp. IMAPS) in at least $96.19 \%$ (resp. 89.35\%) of the cases (Tables X and XI)

In addition, as part of our passive data collection, we also measured which authentication methods servers offered. In contrast to our active measurement, we can only record the authentication methods offered before encryption starts, not those after encryption has started. Table XII shows the percentage of servers that offer a certain authentication mechanism as well as the percentage of connections in which a certain authentication mechanism was offered. Table XIII shows the combination of authentication mechanisms offered that we observed.

The results here are not encouraging-while, according to Table XIII, only $68.88 \%$ of servers offer authentication before STARTTLS, $39.87 \%$ of all servers offer only authentication based on PLAIN and LOGIN. When looking at the number of connections, this picture is even more pronounced with only $4.94 \%$ of connections containing information about authentication mechanisms before STARTTLS, but $3.51 \%$ of all observed connections containing only plaintext authentication mecha- 
TABLE XII: Authentication mechanisms observed in connections on SUBMISSION port.

\begin{tabular}{lrr}
\hline \multicolumn{1}{c}{ Mechanism } & Connections & Servers \\
\hline PLAIN & $4.4 \%$ & $39 \%$ \\
LOGIN & $4.3 \%$ & $37 \%$ \\
CRAM-MD5 & $0.7 \%$ & $10 \%$ \\
DIGEST-MD5 & $0.5 \%$ & $3.7 \%$ \\
XAOL-UAS-MB & $0.4 \%$ & $1.8 \%$ \\
GSSAPI & $0.3 \%$ & $4.3 \%$ \\
NTLM & $0.3 \%$ & $3.7 \%$ \\
XOAUTH2 & $0.03 \%$ & $1.2 \%$ \\
XYMCOOKIE & $0.01 \%$ & $0.6 \%$ \\
\hline
\end{tabular}

TABLE XIII: Combinations of authentication mechanisms observed in connections on SUBMISSION port..

\begin{tabular}{lrr}
\hline \multicolumn{1}{c}{ Mechanisms } & Connections & Servers \\
\hline PLAIN, LOGIN & $1.82 \%$ & $20.86 \%$ \\
LOGIN, PLAIN & $1.68 \%$ & $18.40 \%$ \\
LOGIN,PLAIN,XAOL-UAS-MB & $0.19 \%$ & $1.84 \%$ \\
PLAIN,LOGIN,XAOL-UAS-MB & $0.16 \%$ & $1.84 \%$ \\
GSSAPI & $0.11 \%$ & $1.23 \%$ \\
GSSAPI,NTLM & $0.10 \%$ & $1.84 \%$ \\
LOGIN,PLAIN,CRAM-MD5 & $0.09 \%$ & $3.68 \%$ \\
DIGEST-MD5,CRAM-MD5 & $0.09 \%$ & $0.61 \%$ \\
CRAM-MD5,DIGEST-MD5 & $0.09 \%$ & $0.61 \%$ \\
PLAIN,LOGIN,CRAM-MD5 & $0.08 \%$ & $0.61 \%$ \\
\hline Other 17 combinations observed & $1.09 \%$ & $14.68 \%$ \\
\hline
\end{tabular}

nisms. This is consistent with our findings in Section IV-A that showed that $97 \%$ of SUBMISSION connections upgraded their connection using STARTTLS. Nonetheless, $71 \%$ of clients who did not upgrade their connections also used plaintext mechanisms to authenticate.

Moreover, $31 \%$ of observed IMAP servers (serving $16 \%$ of passively observed connections) refused plaintext logins before encryption (with the LOGINDISABLED capability).

\section{RISKS, THREATS, AND MITIGATION}

In this section, we first summarise the current risks and threats to Internet communication protocols, based on our analyses. We also present recommendations on how to improve the situation in the future. For our discussion, it is important to consider which attacker one wishes to defend against: some security configurations are strong enough against passive, eavesdropping attackers. They are thus secure against global pervasive monitoring of traffic: active attacks require much higher effort and can, in all likelihood, not be carried out on global scale yet.

\section{A. STARTTLS semantics}

Many of the discussed communication protocols, and especially SMTP, rely on STARTTLS to initiate encrypted connections. The problem is that, as shown in Section IV-A, less than $51 \%$ of servers support upgrading connections to TLS. Fortunately, some providers pushed strongly for better adoption of TLS in the last years, increasing the share of connections that use TLS by a significant amount. Nonetheless, in the interest of reliability, many clients and servers will fall back to non-encrypted connections should STARTTLS not be offered.

\section{B. Cipher use}

A common problem is the continued choice of weak ciphers in communication protocols. Depending on the protocol, we still see up to $17 \%$ of connections choosing RC4, which has been deprecated in [36]. This is in contrast to the Web at large, where currently about $10 \%$ of connections use RC 4 ciphers, according to the ICSI Certificate Notary. This difference is likely caused by the push to increment Web security in the last few years, where academia, industry, and the open source community have driven adoption of more modern ciphers. This movement does not seem to have reached other, non-web protocols. Another fact that supports this hypothesis is the high number of connections that do not use forward-secure ciphers (more than $30 \%$ for some of the protocols we observed).

Another issue is the use of Diffie-Hellman parameter sizes of 1024 bits or less in more than $\frac{2}{3}$ of all observed connections. While there are some limited cases in which this might be necessary for legacy compatibility ${ }^{20}$, it seems unlikely that this is a conscious choice by server operators.

\section{Certificate chains and their validity}

We showed that a high number of servers, especially as compared to earlier scans of the HTTPS protocol, serve chains using broken or self-signed certificates. This is of particular importance for SMTP with STARTTLS. If servers with unverifiable certificates are actually able to receive email from SSL/TLS-capable servers, this suggests that a significant number of servers are not verifying certificates in outgoing connections or ignore certificate errors. This means that there are likely many cases where SSL/TLS does not provide MitM protection in server-to-server connections, a finding that is also supported by [12]. As noted before, SMTP tends to prioritise delivery before security (for good reason), and operators will likely favour such an approach to ensure messages reach their destination. On a more positive note, our passive data did reveal that, unlike the servers at large, a much larger ratio of the connections we observed used chains that we could verify. We assume this to be due to the fact that large providers are more capable and willing to invest the time in securing their SSL/TLS setups.

\section{Authentication methods}

The more secure challenge-response authentication mechanisms for SMTP and IMAP do not seem to enjoy much popularity: the methods PLAIN and LOGIN are preferred. We speculate this is because operators tend to assume their connections are already secure because of the use of SSL/TLShowever, this is only true if certificates are actually correct and strong ciphers are used. We also found that client authentication methods are sometimes offered before the negotiation of a SSL/TLS session-the vast majority being PLAIN and LOGIN again. This means that an eavesdropper or active MitM can collect the plaintext of specific messages and is also able to acquire login credentials.

\footnotetext{
${ }^{20}$ Java $\leq 7$ cannot use DH parameters larger than 1024 bits; see http://blog. ivanristic.com/2014/03/ssl-tls-improvements-in-java-8.html.
} 


\section{E. Suggestions for improvement}

We offer several actionable recommendations based on our analyses.

a) Observable infrastructure: One key element to improving messaging security is to create awareness. We believe better observability can be provided in a two-pronged approach: through more regular active scans, but also by facilitating observation of the security of one's own communication. Much insight can be gained by security-conscious users by information available on the dashboards mentioned in Section II-E. We believe that current efforts like SSL Pulse, which aims to enhance the use and security of TLS on the Web, should be extended to other communication protocols. The coverage should also be extended to the entire Internet. The data provided by censys.io [8] is a good source. Auditing mechanisms that so far are meant primarily for use on the Web, such as Certificate Transparency by Google, could also be extended to include email or chat servers.

Another way to improve the situation would be for client software to have clearer user warnings when connecting to servers in insecure ways. Unlike the Web, the messaging infrastructure uses intermediate relays. It is, however, possible to derive (partial) information about the path an email has taken or is going to take [12]. User-agents could be extended to report information about this and signal if the used cryptography is deemed secure.

b) Deployability and configuration: The high number of invalid or otherwise unverifiable certificate chains is a serious obstacle to the ubiquitous adoption of STARTTLS for email forwarding. Two of the reasons for this poor deployment are the costs of certification by CAs and the high difficulty of proper server configuration. The Let's Encrypt ${ }^{21}$ initiative, which currently focuses on Web certification, addresses both issues and should be extended to include email and chat. However, configuration complexity is not straight-forward to address. BetterCrypto.org ${ }^{22}$, for example, provides a guide to correct TLS configuration. This approach scales poorly as almost every software suite uses its own configuration format (or so it often seems). We thus call for a standard for unified SSL/TLS configuration: there is no reason why certificates and cryptography cannot be configured using a unified syntax. Configuration files for SSL/TLS could simply be included (or parsed) in the general configuration of a software.

A recent RFC [5] describes how to increase the security of SMTP opportunistically by using the TLSA record of DNS [21] to signal TLS support. This removes CAs as a single point of failure. Due to its opportunistic nature, the approach can be deployed incrementally. It defeats attackers carrying out global pervasive monitoring. However, DNS/DNSSEC is once again a complex system-thus, this approach also calls for better configuration support and a unified configuration syntax.

c) Flag-days for mandatory encryption: Large providers should research the impact of refusing insecure connectionsthis may cause smaller providers to use verifiable chains. To preserve reliability of delivery, providers could deploy a form of grey-listing for senders using insecure connections and only

\footnotetext{
${ }^{21}$ https://letsencrypt.org/

${ }^{22}$ https://bettercrypto.org
}

offer fast message delivery to mail servers capable of using SSL/TLS in a secure fashion.

A broad call for mandatory encryption and enforcing STARTTLS before using a connection could further push the adoption of better security practices. An example showing that community-based actions have a chance of succeeding is $\mathrm{XMPP}^{23}$. This can be combined with a pinning-like approach where clients refuse plaintext connections, especially to popular servers, if previous connections had used STARTTLS. This is in line with the findings of [9], where the authors show that certain ISPs may try to downgrade connections by stripping STARTTLS commands.

A further step to foster the proper use of SSL/TLS could be taken by package and operating system maintainers. If packages shipped with a safe, modern configuration for the ciphers to use, many of the problems we highlighted could be remedied. A summary of the best current practices for this purpose can be found in [43]. Conversely, application packages should never be shipped with default cryptographic material, not even as examples. A saner approach would be to provide scripts to generate the keys and certificates.

d) Application-layer authentication: Challenge-response authentication mechanisms like CRAM and SCRAM avoid sending credentials over the wire. SCRAM even allows servers to store the password in non-plaintext form, thus combining improved credential storage with safer authentication. Support for challenge-response forms of authentication seems to be lacking, possibly because developers see no need for it and operators fear the complexity. Standard packages and unified configuration can help here.

\section{CONCLUSION}

This paper presented the largest study of the security of the standard Internet messaging infrastructure to date. Based on active scans of servers and passive monitoring of client connections, we collected the parameters used to establish SSL/TLS sessions, the details of X.509 certificates offered by servers, and the application-layer authentication methods offered to, and used by, clients. Across the whole Internet, we found a worryingly high number of poorly secured servers. This was either due to cryptographic parameter and cipher choices or due to invalid or duplicated cryptographic material. Too many servers also offer weak application-layer authentication methods. A silver lining is that there are significantly better deployments in the most popular services, and a majority of observed clients connected using reasonably secure parameters when they did request encryption. Nonetheless, too many of the connections we observed were still performed in the clear. Moreover, we found that many client-to-server setups, especially for SMTP, did not use valid credentials. This means that email in transit may often be delivered over unencrypted and unauthenticated hops. We gave a list of recommendations that are actionable and can help to significantly improve the situation.

\section{ACKNOWLEDGMENTS}

This work was supported by the National Science Foundation under grant numbers CNS-1528156 and ACI-1348077.

\footnotetext{
${ }^{23}$ http://blog.prosody.im/mandatory-encryption-on-xmpp-starts-today/
} 
Any opinions, findings, and conclusions or recommendations expressed in this material are those of the author(s) and do not necessarily reflect the views of the NSF.

\section{REFERENCES}

[1] D. Akhawe et al. "Here's My Cert, So Trust Me, Maybe? Understanding TLS Errors on the Web". In: Proc. Int. World Wide Web Conference. 2013.

[2] J. Amann et al. "No Attack Necessary: the Surprising Dynamics of SSL Trust Relationships". In: Ann. Computer Security Applications Conference (ACSAC). 2013.

[3] Baseline requirements for the issuance and management of publicly-trusted certificates, v.1.0. CA/Browser Forum, 2011.

[4] M. Crispin. Internet Message Access Protocol - Version 4rev1. RFC 3501 (Proposed Standard). IETF, Mar. 2003.

[5] V. Dukhovni and W. Hardaker. SMTP Security via Opportunistic DNS-Based Authentication of Named Entities (DANE) Transport Layer Security (TLS). RFC 7672 (Proposed Standard). IETF, 2015

[6] Z. Durumeric, E. Wustrow, and J. A. Halderman. "ZMap: Fast Internet-wide Scanning and Its Security Applications”. In: Proc. USENIX Security. 2013.

[7] Z. Durumeric et al. "Analysis of the HTTPS certificate ecosystem". In: Proc. ACM SIGCOMM Internet Measurement Conference (IMC). 2013

[8] Z. Durumeric et al. "Censys-A Search Engine Backed by Internet-Wide Scanning". In: Proc. ACM Computer and Communications Security (CCS). 2015.

[9] Z. Durumeric et al. "Neither Snow Nor Rain Nor MITM... An Empirical Analysis of Mail Delivery Security". In: Proc. ACM SIGCOMM Internet Measurement Conference (IMC). 2015.

[10] Z. Durumeric et al. "The Matter of Heartbleed". In: Proc. ACM SIGCOMM Internet Measurement Conference (IMC). 2014.

[11] Email Statistics Report, 2014-2018. The Radicati Group, Inc. URL: http://www.radicati.com/wp/wp-content/uploads/2014/01/ Email-Statistics-Report-2014-2018-Executive-Summary.pdf.

[12] I. Foster et al. "Security by Any Other Name: On the Effectiveness of Provider Based Email Security". In: Proc. ACM Computer and Communications Security (CCS). 2015.

[13] A. Freier, P. Karlton, and P. Kocher. The Secure Sockets Layer (SSL) Protocol Version 3.0. RFC 6101 (Historic). IETF, Aug. 2011.

[14] O. Gasser, R. Holz, and G. Carle. "A deeper understanding of SSH: results from Internet-wide scans". In: Proc. Network Operations and Management Symposium (NOMS). 2014.

[15] R. Gellens and J. Klensin. Message Submission. RFC 2476 (Proposed Standard). IETF, Dec. 1998.

[16] R. Gellens and J. Klensin. Message Submission for Mail. RFC 6409 (Internet Standard). IETF, Nov. 2011.

[17] R. Hartmann. Default Port for Internet Relay Chat (IRC) via TLS/SSL. RFC 7194 (Informational). IETF, Aug. 2014.

[18] J. Heidemann et al. "Census and survey of the visible Internet". In: Proc. ACM SIGCOMM Internet Measurement Conference (IMC). 2008.

[19] N. Heninger et al. "Mining Your Ps and Qs: detection of Widespread Weak Keys in Network Devices". In: Proc. USENIX Security. 2012.

[20] P. Hoffman. SMTP Service Extension for Secure SMTP over Transport Layer Security. RFC 3207 (Proposed Standard). IETF, Feb. 2002.

[21] P. Hoffman and J. Schlyter. The DNS-Based Authentication of Named Entities (DANE) Transport Layer Security (TLS) Protocol: TLSA. RFC 6698 (Proposed Standard). IETF, Aug. 2012.

[22] P. Hoffman. SMTP Service Extension for Secure SMTP over $T L S$. Internet-Draft draft-hoffman-smtp-ssl-04.txt. 1999.
[23] R. Holz. "Empirical analysis of Public Key Infrastructures and investigation of improvements". PhD thesis. Technical University of Munich, 2014.

[24] R. Holz et al. "The SSL landscape - a thorough analysis of the X.509 PKI using active and passive measurements". In: Proc. ACM SIGCOMM Internet Measurement Conference (IMC). 2011.

[25] L.-S. Huang et al. "An Experimental Study of TLS Forward Secrecy Deployments". In: Internet Computing, IEEE 18.6 (2014).

[26] C. Kalt. Internet Relay Chat: Server Protocol. RFC 2813 (Informational). IETF, Apr. 2000.

[27] J. Klensin. Simple Mail Transfer Protocol. RFC 5321 (Draft Standard). IETF, Oct. 2008.

[28] J. Klensin, R. Catoe, and P. Krumviede. IMAP/POP AUTHorize Extension for Simple Challenge/Response. RFC 2195 (Proposed Standard). IETF, Sept. 1997.

[29] D. Leonard and D. Loguinov. "Demystifying service discovery: Implementing an Internet-wide scanner". In: Proc. ACM SIGCOMM Internet Measurement Conference (IMC). 2010.

[30] J. Myers and M. Rose. Post Office Protocol - Version 3. RFC 1939 (INTERNET STANDARD). IETF, May 1996.

[31] M. Myers and H. Tschofenig. Online Certificate Status Protocol (OCSP) Extensions to IKEv2. RFC 4806 (Proposed Standard). IETF, Feb. 2007.

[32] C. Newman. Using TLS with IMAP, POP3 and ACAP. RFC 2595 (Proposed Standard). IETF, June 1999.

[33] C. Newman et al. Salted Challenge Response Authentication Mechanism (SCRAM) SASL and GSS-API Mechanisms. RFC 5802 (Proposed Standard). IETF, July 2010.

[34] J. Oikarinen and D. Reed. Internet Relay Chat Protocol. RFC 1459 (Experimental). IETF, May 1993.

[35] V. Paxson. "Bro: A System for Detecting Network Intruders in Real-Time". In: Computer Networks 31.23-24 (1999).

[36] A. Popov. Prohibiting RC4 Cipher Suites. RFC 7465 (Proposed Standard). IETF, Feb. 2015.

[37] N. Provos and P. Honeyman. "ScanSSH—scanning the Internet for SSH servers". In: Proc. USENIX LISA. 2001.

[38] I. Ristić. Bullet-proof SSL and TLS. Feisty Duck Ltd., 2014.

[39] P. Saint-Andre. Extensible Messaging and Presence Protocol (XMPP): Core. RFC 6120 (Proposed Standard). IETF, Mar. 2011.

[40] P. Saint-Andre. Extensible Messaging and Presence Protocol (XMPP): Instant Messaging and Presence. RFC 6121 (Proposed Standard). IETF, Mar. 2011.

[41] P. Saint-Andre and T. Alkemade. Use of Transport Layer Security (TLS) in the Extensible Messaging and Presence Protocol (XMPP). RFC 7590 (Proposed Standard). IETF, June 2015.

[42] J. Schlamp et al. "Investigating the Nature of Routing Anomalies: Closing in on Subprefix Hijacking Attacks". In: Proc. 7th Int. Workshop on Traffic Monitoring and Analysis. 2015.

[43] Y. Sheffer, R. Holz, and P. Saint-Andre. Recommendations for Secure Use of Transport Layer Security (TLS) and Datagram Transport Layer Security (DTLS). RFC 7525 (Best Current Practice). IETF, May 2015.

[44] Y. Sheffer, R. Holz, and P. Saint-Andre. Summarizing Known Attacks on Transport Layer Security (TLS) and Datagram TLS (DTLS). RFC 7457 (Informational). IETF, Feb. 2015. URL: http://www.ietf.org/rfc/rfc7457.txt.

[45] N. Vratonjic et al. "The inconvenient truth about Web certificates". In: Proc. Workshop on Economics in Information Security. 2011.

[46] K. Zeilenga. The PLAIN Simple Authentication and Security Layer (SASL) Mechanism. RFC 4616 (Proposed Standard). IETF, Aug. 2006. 